\title{
Ernährungstherapie bei Allergien
}

\author{
Autorin: Monika Pirlet-Gottwald
}

\section{ZUSAMMENFASSUNG}

Der Entstehungsort einer allergischen Reaktion und das Symptomorgan liegen oft weit auseinander. Die orale Aufnahme eines Allergens kann z. B. Hautreaktionen verursachen oder verstärken. Irritationen des inflammatorischen Systems können zusätzlich das Auftreten oder die Ausprägung einer Allergie beeinflussen.

Kaum ein anderes therapeutisches Verfahren greift so grundlegend und tief in den Genesungsprozess ein wie die Sanierung des Verdauungssystems. Wenn die intestinale Intoxikation und bakterielle Dyspepsie ausheilen, kommen das Immunsystem und bestehende entzündliche Reaktionen zur Ruhe.

Der Artikel erläutert das therapeutische Vorgehen und die möglichen Erfolge anhand von Fällen aus der Praxis.

\section{ABSTRACT}

The point of origin of an allergic reaction and the symptom organ are often far apart. The oral intake of an allergen can cause or intensify skin reactions for example. Irritations of the inflammatory system can additionally influence the occurrence or the manifestation of an allergy. There is hardly another therapeutic procedure, which interferes so fundamentally and deeply with the recovery process, as it is the case with restoring the digestive system. When the intestinal intoxication and the bacterial dyspepsia are healing, the immune system and the existing inflammatory reactions come to rest.

The article explains the therapeutic procedure as well as the possible success whit the help of cases from practice.
Schlüsselwörter
Allergie, Ernährungstherapie, intestinales Immunsystem.
Keywords

Allergy, nutritional therapy, intestinal immune system.

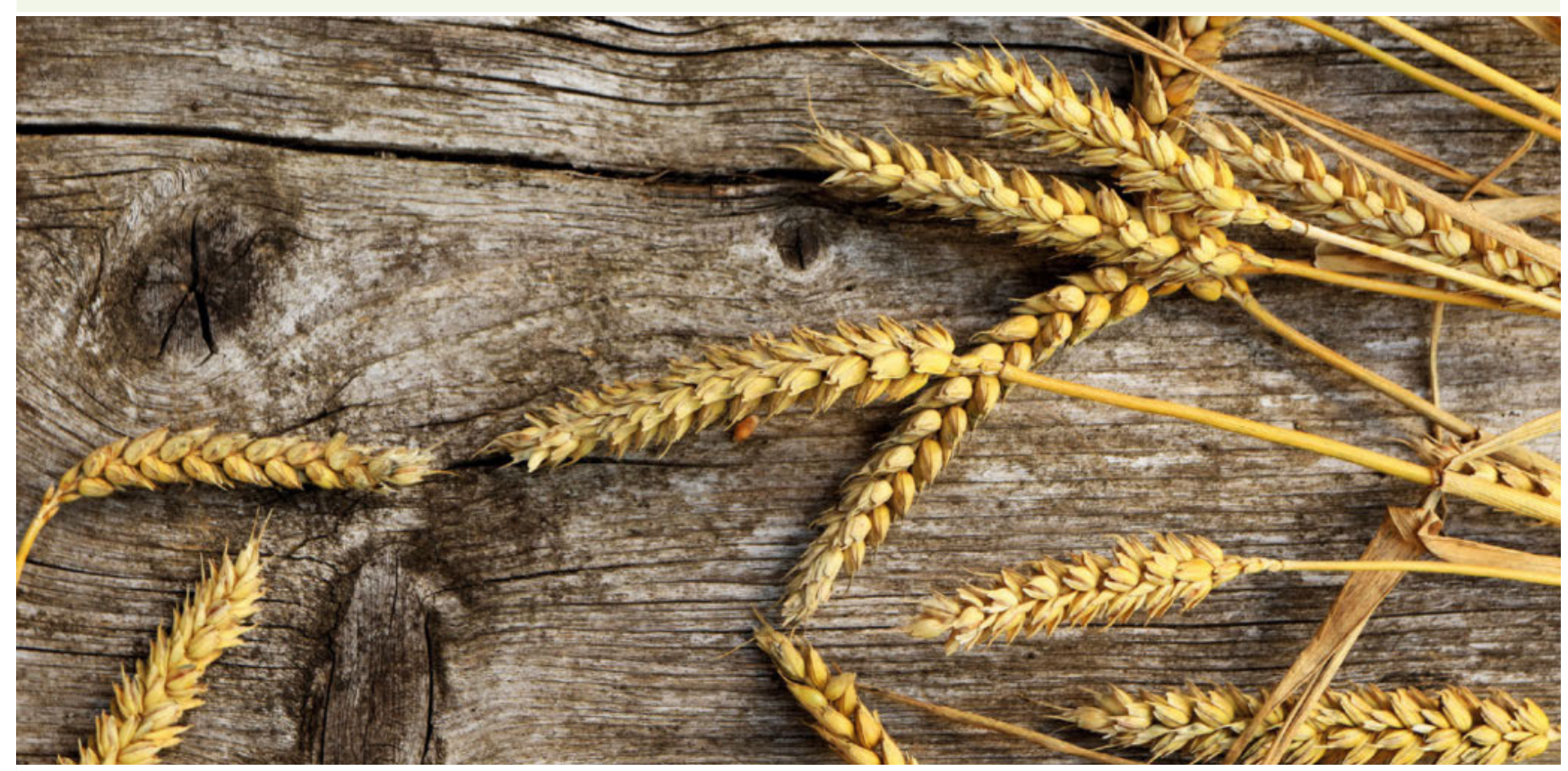

Abb. 1 Das in Getreiden wie Weizen enthaltene Gluten gehört zu den häufigsten Allergenen aus Nahrungsmitteln. @ nightsphotos/Adobe Stock

Allergien sind weit verbreitet. Bei manchen Menschen ist es eine bestimmte Pollenart, bei anderen ein Nahrungsmittel und wieder andere reagieren auf das Gift einer Biene allergisch: Fremdproteine werden für einen Organismus dann zu „Allergenen“, wenn sie aufgrund eines Integritätsverlusts der Schleimhaut in die tieferen Mukosaschichten eindringen, dort als fremde „Eindringlinge“ vom Immunsystem erkannt und mit einer chronisch verlaufenden Entzündungs- und Abwehrreaktion bekämpft werden. Diese allergischen Reaktionen liegen meist in der Haut oder den Schleimhäuten: die atopische Reaktion der Neurodermitis, Rhinitis, Konjunktivitis, Bronchitis, Sinusitis sowie die unterschiedlichen Nahrungsmittelallergien in der intestinalen Schleimhaut.

Traditionelle organspezifische Therapieansätze bemühen sich, die inflammatorischen und immunologischen Reaktionen in dem betroffenen Areal zu behandeln. Doch grei- 
fen solche Behandlungsweisen zu kurz, denn die Schleimhäute sind eng über das Blut- und das mukosale Immunund Lymphsystem miteinander verbunden. Zudem liegen der Entstehungsort einer allergischen Reaktion und das Symptomorgan oftmals weit auseinander: So kann die orale Aufnahme eines Allergens die entzündlichen Reaktionen in der Haut (z. B. die Neurodermitis) verursachen oder verstärken. Zusätzlich beeinflussen alle anderen Irritationen des inflammatorischen Systems wie Infekte und eine Imbalance des vegetativen Nervensystems oder chronisch entzündliche Erkrankungen wie der M. Crohn das Auftreten oder die Ausprägung allergischer Reaktionen.

\section{Das intestinale Immunsystem und Allergien}

Eine ganz besondere Bedeutung kommt dem intestinalen Immunsystem zu („gut associated lymphoid tissue“ (GALT)), denn es macht den größten Teil unseres Immunsystems aus. $80 \%$ aller Immunzellen haben hier in den Peyer'schen Plaques, in der Mesenterialwurzel ihren Ursprung. Kein anderes Organ bildet ähnlich viele Antikörper: ein fein abgestimmtes System aus Makrophagen, Mastzellen, B- und T-Lymphozyten, zellulären und humoralen Immunreaktionen. Fremdproteine aus der Nahrung werden hier für einen Organismus zu „Allergenen“, wenn sie nicht ordnungsgemäß verdaut, d. h. enzymatisch in Aminosäuren zerlegt werden und sie aufgrund eines Integritätsverlusts der Schleimhaut in die tieferen Mukosaschichten eindringen.

Nun zählen Verdauungsstörungen zu den häufigsten Beschwerden unserer Zeit. Veränderte Nahrungsmittel, zu kurze und unvollständige Zubereitungen der Nahrung und veränderte Lebensgewohnheiten beeinflussen die Verdauungsleistung. Hektik und hastiges Essen verhindern das sorgfältige Kauen, das richtige Einspeicheln, das Aus-Schmecken, Genießen und Aus-Kosten der Nahrung. Zu große Einzelmahlzeiten, zu viel oder zu vielerlei, mangelnde Azidität des Magens (Cave: Antazida) oder unzureichende Enzymaktivität im Magen oder Pankreas verhindern die fermentative Aufschlüsselung der Proteine. Auch sind viele Nahrungsmittel zu schwer verdaulich: rohe Körner, grobes Vollkornbrot, Salate, Fett-Gebratenes. Dies gilt insbesondere, wenn sie erst am späteren Abend gegessen werden. Blähungen, wechselnde Stuhlgänge, Nahrungsmittelunverträglichkeiten, ein „gereizter“ Darm sind die ersten und häufigsten Symptome.

Bleibt die Nahrung unverdaut, wird sie von den Bakterien in tieferen Darmabschnitten zersetzt. Faserreiche Kohlenhydrate gären, es entstehen Methanol, Butanol, Propanol und andere Fuselalkohole und Gase: erkennbar am aufgetriebenen Leib und Blähungen.
Proteine oder Polypeptide werden durch proteolytische Keime zersetzt, sie faulen. Es entstehen Ammoniak, vermehrt biogene Amine wie Tyramin oder Kadaverin und toxische Abbauprodukte wie Indol, Kresol, Phenol, Skatol. Diese sind für die Pseudoallergien verantwortlich zu machen. Sie lösen Entzündungsreaktionen in der Schleimhaut aus, die Tight-junctions der Darmschleimhautzellen lösen sich, wir sprechen vom Leaky-gut-Syndrom, dem Syndrom des „löchrigen Darms“.

\section{Die Schleimhautintegrität ist gestört. Fremdeiweiße können ungehindert die Grenzfläche penetrieren.}

Eine nachhaltige Therapie allergischer Erkrankungen muss daher den Darm immer mit behandeln, denn:

\section{„Egal wie der Vater der Krankheit heißt, die Mutter ist immer die Ernährung. “}

(chinesisches Sprichwort)

Eine nicht unerhebliche Bedeutung kommt auch dem vegetativen Nervensystem zu: Es durchzieht in einem komplexen Netzwerk das gesamte mesenchymale Bindegewebe (Plexus submucosus und Plexus myentericus) bis an die Epithelschicht heran und verknüpft so Haut und Schleimhaut mit dem inneren Milieu. Hier vernetzen sich Parasympathikus und Sympathikus, hier werden etwa $90 \%$ des regulierenden Hormons Serotonin gebildet. Die intestinale vegetative Balance steht in engem Zusammenhang mit den neuralen und inflammatorischen Regulationen in der Haut und den übrigen Schleimhautorgansystemen. Störungen in der epithelialen Schleimhaut oder chronische auch stille Entzündungen (low grade inflammations) der Mukosa führen zu Irritationen dieses vegetativen Nervensystems, zur Einschränkung der mikrozirkulatorischen Versorgung und Trophik der Schleimhaut und Mukosa und oftmals zur Chronifizierung der entzündlichen Reaktionen. Dies erklärt die häufigen Exazerbationen von Allergien unter Stressbelastungen.

\section{Mikrobiota}

Nirgendwo sonst im Körper findet ein intensiverer Kontakt mit fremden Stoffen statt, als im Darm. Deshalb besiedelt eine äußerst leistungsstarke Schutzbarriere in Form von gesunden Bakterien unseren Darm. Es ist eine komplexe, feinabgestimmte Bakterienflora (Mikrobiota), die immer mehr in den Fokus der Diagnostik und Therapie tritt. Im Jejunum ist die Kolonisation eher niedrig und es überwiegt die anaerobe Flora im eher sauren Milieu aus Lactobazillen und Bifidobakterien, aber auch wenige anaerobe Enterokokken sitzen hier. Im Ileum verschiebt sich die Flora in den alkalischen Bereich mit der vermehrten Besiedelung durch anaerobe Keime (Enterokokken, Escherischia coli). Die Konstanz der Milieubedingungen, ein hochwertiges Angebot und die vollständige Aufschlüsselung der zugeführten Nahrung begünstigen eine gesunde komplexe 


\begin{tabular}{|c|c|c|c|c|}
\hline \multicolumn{5}{|c|}{ Nachweis von Bakterien aerob } \\
\hline Escherichia coli & $6,0 \times 10^{\wedge} 4$ & KBE/g Stuhl & $10^{\wedge} 6-10^{\wedge} 7$ & $\begin{array}{l}\mathrm{Fe} \\
\text { A) KULTAZ }\end{array}$ \\
\hline Escherichia coli Biovare & $<1,0 \times 10^{\wedge} 4$ & KBE/g Stuhl & $<1,0 \times 10^{\wedge} 4$ & $\begin{aligned} \mathrm{Fe} \\
\text { A) KULAZ }\end{aligned}$ \\
\hline Proteus species & $<1,0 \times 10^{\wedge} 4$ & KBE/g Stuhl & $<1,0 \times 10^{\wedge} 4$ & $\begin{aligned} \mathrm{Fe} \\
\text { A) KUTAZ }\end{aligned}$ \\
\hline Klebsiella species & $<1,0 \times 10^{\wedge} 4$ & KBE/g Stuhl & $<1,0 \times 10^{\wedge} 4$ & $\begin{aligned} \mathrm{Fe} \\
\text { A) KULTAZ }\end{aligned}$ \\
\hline Pseudomonas species & $<1,0 \times 10^{\wedge} 4$ & KBE/g Stuhl & $<1,0 \times 10^{\wedge} 4$ & $\begin{aligned} \mathrm{Fe} \\
\text { A) KULTAZ }\end{aligned}$ \\
\hline Enterobacter species & $<1,0 \times 10^{\wedge} 4$ & KBE/g Stuhl & $<1,0 \times 10^{\wedge} 4$ & $\begin{aligned} \mathrm{Fe} \\
\text { A) KULTAZ }\end{aligned}$ \\
\hline Serratia species & $<1,0 \times 10^{\wedge} 4$ & KBE/g Stuhl & $<1,0 \times 10^{\wedge} 4$ & $\begin{aligned} \mathrm{Fe} \\
\text { A) KULTAZ }\end{aligned}$ \\
\hline Hafnia species & $<1,0 \times 10^{\wedge} 4$ & KBE/g Stuhl & $<1,0 \times 10^{\wedge} 4$ & A) KULTAZ \\
\hline Enterococcus species & $<1,0 \times 10^{\wedge} 4$ & KBE/g Stuhl & $10^{\wedge} 6-10^{\wedge} 7$ & $\begin{aligned} & \mathrm{Fe} \\
& \text { A) KULAZ }\end{aligned}$ \\
\hline \multicolumn{5}{|c|}{ Nachweis von Bakterien anaerob } \\
\hline Bifidobakterium species & $6,0 \times 10^{\wedge} 8$ & KBE/g Stuhl & $10^{\wedge} 9-10^{\wedge} 11$ & 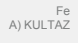 \\
\hline Bacteroides species & $2,0 \times 10^{\wedge} 8$ & KBE/g Stuhl & $10^{\wedge} 9-10^{\wedge} 11$ & $\begin{aligned} \mathrm{Fe} \\
\text { A) KULTAZ }\end{aligned}$ \\
\hline Lactobacillus species & $<1,0 \times 10^{\wedge} 4$ & KBE/g Stuhl & $10^{\wedge} 5-10^{\wedge} 7$ & $\begin{aligned} \mathrm{Fe} \\
\text { A) KULTAZ }\end{aligned}$ \\
\hline Clostridium species & $<1,0 \times 10^{\wedge} 5$ & KBE/g Stuhl & $<1,0 \times 10^{\wedge} 5$ & $\begin{array}{l}\mathrm{Fe} \\
\text { A) KULTAZ }\end{array}$ \\
\hline
\end{tabular}

Abb. 2 Gastroenterologischer Laborbefund von Marie W.

\begin{tabular}{|c|c|c|c|c|}
\hline Zonulin & 60,77 & $\mathrm{ng} / \mathrm{ml}$ & $<55$ & $\begin{aligned} \mathrm{Fe} & -1 \\
\text { A) ELSA } & \end{aligned}$ \\
\hline \multicolumn{5}{|c|}{ Spezielle gastroenterologische Diagnostik } \\
\hline \multicolumn{5}{|l|}{ Glutensensitive Enteropathie / Zöliakie } \\
\hline Anti-Transglutaminase AK i. Stuhl & $<50$ & $\mathrm{U} / \mathrm{I}$ & $<100$ & $\begin{aligned} \text { Fe } & \\
\text { A) ELSA } & \end{aligned}$ \\
\hline
\end{tabular}

Abb. 3 Spezielle gastroenterologische Diagnostik von Marie W.

Bakterienpopulation. Diese erfüllen für den Menschen lebenswichtige Funktionen:

- Verdauung (Aufschlüsselung der Nahrung, Fermentation, Enzymproduktion) - insbesondere die Lactobazillen und Bifidobakterien

- Synthese der Vitamine $B_{6}, B_{11}, B_{12}$ und $K$

- Verringerung des Säuregrads im Dickdarm (Resorption der Mineralien)

- Produktion von kurzkettigen Fettsäuren, Butyraten (für die Darmperistaltik und als Energielieferant der Darmschleimhautzellen)

- Bindung der Gallensäuresalze (Cholesterinmetabolismus)

- Immunmodulation - v. a. E.-coli-Bakterien

- Enterococcus faecalis stimuliert die Produktion und Freisetzung von slgA

\section{Diagnostik}

Als erste diagnostische Methoden dienen uns der visuelle Eindruck der Bauchform sowie die manuelle Untersuchung der Bauchorgane, des Spannungszustandes und der Mesenterialwurzel. Vermehrte Gasbildung, Spannungen und Druckdolenzen über dem Bauchraum deuten auf entzündliche Veränderungen im gastrointestinalen Organsystem hin.
Eine Untersuchung des Stuhls kann Aufschluss über die Entzündungssituation der Darmschleimhaut geben: $\alpha$-1-Antitrypsin und Calprotectin. Immunglobulin A ( $\lg \mathrm{A}$ ) wird in den Epithelzellen als Abwehrprotein sezerniert. Zonulin wird beim Auflösen der Tight Junctions der Schleimhautzellen vermehrt in das Darmlumen abgegeben und im Stuhl ausgeschieden und dient daher zum Nachweis des Leaky gut.

Immunreaktionen gegen penetrierende Nahrungsproteine können im Serum nachgewiesen werden. Hier nehmen viele Allergien ihren Anfang: Urtikaria, Ekzeme, Neurodermitis, Kuhmilchallergien, Ei-Eiweiß-Allergien. Neben den IgE-vermittelten allergischen Sofortreaktionen sind die Immunglobuline $G$ für verzögerte Reaktionen verantwortlich zu machen: Kopfschmerzen, Gelenkbeschwerden, aber auch als Verstärker (Immuntrigger) für allergische Reaktionen.

Histamin und andere biogene Amine (wie Tyramin) können im Serum, aber auch im Stuhl spezifisch für die intestinale Bildung nachgewiesen werden. Sie deuten auf vermehrte Fäulnisreaktionen im Darm hin.

Das Eosinophile Protein X (EPX) ist ein einfacher Marker für die Entzündungsprozesse bei Nahrungsmittelallergien und -unverträglichkeiten. Eosinophile Granulozyten spie- 
len eine Schlüsselrolle im asthmatisch-allergischen Entzündungsgeschehen. Das EPX ist ein guter Suchtest insbesondere bei Kindern zur Überprüfung der Beteiligung von Nahrungsmitteln bei subklinischen Entzündungsreaktionen. So können erfolgreiche Eliminationsdiäten anhand des EPX verfolgt werden.

\section{Patientenfälle aus der Praxis}

\section{Marie W. 10 Jahre}

Anamnese: Neurodermitis seit dem dritten Lebensmonat. Häufig Müdigkeit und migräneartige Kopfschmerzen. Die Mutter berichtet eine normal verlaufene Schwangerschaft und Geburt, 4 Monate voll gestillt, anschließend hypoallergene Flaschennahrung und Aufbau zur Familienernährung. Diese besteht hauptsächlich aus Gemüse und Rohkost, Getreideflocken/Müsli, Mischbrot mit vegetarischen Brotaufstrichen, Sojaprodukten, wenig Schaf- oder Ziegenkäse. Die Mutter lebt vegetarisch bis vegan und will kein Fleisch und keinen Fisch kochen bzw. zubereiten.

Labor: IgE-vermittelte Allergie auf Ei-Eiweiß sehr hoch, auf Kuhmilch mäßig.

Diagnostik: leicht übergewichtige Patientin, teigig pastöses Gesicht, sehr weiches Bindegewebe, Bauch gebläht und deutlich schmerzhaft bei der Palpation. Dünndarm nicht abgrenzbar. Stuhlgang täglich, schmierig geformt, stinkend. Blähungen sehr unangenehm riechend.

Wenn sie nur ganz wenig Ei isst (z. B. auf Kindergeburtstagen im Kuchen) bekommt sie sofort starke Hautrötungen mit quälendem Juckreiz. Auf Kuhmilch nicht ganz so stark.

Stuhldiagnostik: Deutliche Mangelbesiedelung der immunkompetenten und Verdauungskeime (- Abb. 2). Wenig Entzündungszeichen: normales $\alpha$-1-Antitrypsin und Calprotectin.

Doch deutlich auch eine Antikörperbildung gegen Gliadine ( Abb. 3)!

\section{Lisa P. 52 Jahre}

Anamnese: Seit mehreren Jahren auftretende Urtikaria: Flushartige Rötungen zum Teil mit Quaddelbildung im Gesicht und am Dekolleté. Stark juckend ohne Allgemeinsymptome, ohne Atembeschwerden. Allergie bekannt auf Birkenpollen und Gräser. Die medikamentöse Behandlung mit Antihistaminika bringt nur wenig Besserung. Die Patientin klagt über starke, auch stinkende Blähungen.

Auch die strikte Karenz histaminhaltiger und histaminausschüttender Lebensmittel (Alkohol, altgereifte Käsesorten, Räucherfleisch/-fisch ...) bringt keine nachhaltige Besserung: „Ich weiß gar nicht mehr was ich essen darf! Jetzt esse ich doch schon low carb - auch das hat nicht geholfen!“ 
Obstipation, wechselnd mit plötzlichen Diarrhöen.

\section{Ernährungsanamnese:}

- morgens: Tee, Müsli aus Erdmandel-, Hirse- und Haferflocken mit Wasser und Quark.

- vormittags: 1 Apfel, eine Banane und evtl. saisonales Obst

- mittags (meist auswärts): Salat, Gemüse mit Fleisch oder Fisch

- abends: gemischter Salat mit Schafskäse, Joghurt oder auch Fleisch

Untersuchung: Normalgewichtige Patientin, stark gurgelnde Darmgeräusche, aufgeblähter Leib. Dünndarm stark verspannt und druckschmerzhaft, Mesenterialwurzel mit Abwehrspannung tastbar.

Labor: Histaminausscheidung im Stuhl erhöht. Die Diaminooxidase im Blut lag in der Norm.

\section{Silke F. 43 Jahre, verheiratet zwei Kinder}

Anamnese: Seit mehreren Jahren rezidivierendes trockenes Ekzem an den Handinnenflächen mit tiefen Rissen. Die vom Hautarzt verordneten Kostisonsalbenbehandlungen bringen nur vorübergehend Besserung.

Ernährungsanamnese: „Vollwertig und gesund“

- morgens: Haferflocken/Müsli in Wasser eingeweicht, roh mit Joghurt und geriebenem Apfel

- mittags: Gemüse, Reis, Salate mit Käse, mal ein Ei

- zwischendurch: 1-2 Obst, mal Kekse

- abends: mal Fisch, Salat, Vollkornbrot mit Schaf- oder Ziegenkäse

Eine eigenständige Umstellung vor 3 Jahren auf eine vegane Ernährung brachte keine Besserung, wegen zunehmender Müdigkeit und Schwäche fügte die Patientin wieder zweimal wöchentlich Fisch und täglich Milchprodukte in den Ernährungsplan ein.

Ernährungsmedizinische Diagnostik: Deutlicher Blähbauch, verspannter druckdolenter Dünndarm, Mesenterialwurzel verdickt, mit Abwehrspannung tastbar, Gase deutlich auskultierbar. Immer mal wieder weiche, breiige Stuhlgänge.

Die Frage nach der Esskultur beantwortet die Patientin mit: „Ich bin ein Schnell-Esser und Schlinger.“

Stuhluntersuchung: erhöhte Entzündungswerte: $\alpha-1-A n-$ titrypsin und Zonulin. Darmdysbiose mit einer Vermehrung von aeroben Fäulniskeimen und einer Verminderung der Immunflora Escherichia coli, der Lactobazillen und Bifidobakterien.

Allergiediagnostik: keine Histaminreaktion, keine lgE-Allergie nachweisbar, IgG-Unverträglichkeit auf Kasein und
Lactglobuline der Kuh RAST-Klasse 5, Haselnuss RAST 4, Glutenhaltige Getreide RAST 2.

\section{Der therapeutische Weg}

1) „Feste Nahrung sollst du trinken - flüssige Nahrung sollst du essen." (Hippokrates):

Alle Nahrung muss so gegessen und gekaut werden, dass sie im Mund vollständig verspeichelt ist. Was nicht gekaut werden kann, sollte nicht geschluckt werden. Nur so kann sie im Magen von der Magensäure eingesaftet, im Duodenum von den Enzymen durchdrungen und in ihre Bausteine - Proteine in Aminosäuren und Dipeptide, Kohlenhydrate in Di- und Monosaccharide und Fette in die einzelnen Fettsäuren - zerlegt werden. Nur so ist die Resorption im oberen Dünndarm möglich und Ileum und Colon sind von der Verdauung entlastet. Die Intoxikation kommt zur Ruhe: Kein Leaky gut - keine Aufnahme höhermolekularer Eiweiße - keine Immunreaktionen.

2) „Der Mensch lebt nur von der Hälfte dessen, was er isst, von der anderen leben die Ärzte.“ (Ägypten 2000 v. (hr.):

Also: Kleinere Mahlzeiten und leicht verdauliche Lebensmittel! Zwischen die Hauptmahlzeiten sollten kleine Zwischenmahlzeiten eingeschoben werden. Kleine Nahrungsmengen können besser verdaut werden. Faserhaltige Nahrungsmittel wie Rohkost, Vollkorn oder gar Müsli müssen gemieden werden. Unser Verdauungssystem bildet keine Enzyme (Zellulasen), um die Zellhüllen aufzubrechen. Die Zellulosehülle behindert aber das Eindringen der Verdauungssäfte in die Pflanzenzelle, die in der Zelle eingelagerten Nahrungsstoffe können nur sehr langsam herausgelöst werden.

3) Verdauliche Zubereitung der Nahrungsmittel: Unsere traditionelle Küche hat viele Wege gefunden, dass unsere Nahrung leicht und gut verdaut wird:

- Fleisch kurz braten und lange garen. Markknochen(suppen) stunden- oder tagelang kochen. Alle Nährstoffe werden so herausgelöst, Proteine zerlegt und für die Verdauungssäfte zugänglich gemacht: „Es zergeht auf der Zunge“ - ein hohes Lob an die Küche.

- Reis, Kartoffeln und Gemüse müssen so gargekocht werden, dass sie sich von der Zunge am Gaumen zerdrücken lassen.

- Fleisch, Fisch, Geflügel müssen zart und mürbe werden, dass sie eben „auf der Zunge zergehen“ und sich feinstens zerkauen lassen. Kein rohes Fleisch oder rohen Fisch essen. Ein gekochtes Ei soll zäh-weich sein; Rührei mit etwas Sahne zubereiten - es soll weich und gallertig sein.

- Toast und Zwieback sind deshalb leicht verdaulich, weil sie sich im Mund gut zerkleinern lassen, und weil sie sich schon im Mund mit fermenthaltigem Speichel vollsaugen. 


\begin{tabular}{|c|c|c|c|c|c|c|c|c|c|c|c|c|c|c|}
\hline \multicolumn{15}{|c|}{ IgG4 Nahrungsmittelscreen } \\
\hline \multirow[t]{2}{*}{ Test } & \multirow{2}{*}{$\begin{array}{c}\text { Ergebnis } \\
\mathrm{kU} / \mathrm{l}\end{array}$} & \multicolumn{5}{|c|}{ Normbereich } & \multirow[t]{2}{*}{ Test } & \multirow{2}{*}{$\begin{array}{c}\text { Ergebnis } \\
\text { kU/I }\end{array}$} & \multicolumn{6}{|c|}{ Normbereich } \\
\hline & & 1 & 2 & 3 & 4 & \begin{tabular}{l|l}
5 & 6
\end{tabular} & & & 1 & 2 & 3 & 4 & 5 & 6 \\
\hline \multicolumn{7}{|c|}{ Getreide glutenhaltig } & \multicolumn{8}{|l|}{ Milchprodukte } \\
\hline Dinkel & 90,16 & \multicolumn{5}{|l|}{5} & Kasein & $>100,00$ & \multicolumn{6}{|l|}{6} \\
\hline Hafer & 17,21 & \multicolumn{5}{|l|}{3} & Milchpool & 94,64 & \multicolumn{5}{|l|}{5} & \\
\hline Roggen & 57,97 & \multicolumn{5}{|l|}{5} & \multicolumn{8}{|l|}{ Gemüse, Salate } \\
\hline Weizen & 99,92 & \multicolumn{5}{|l|}{5} & Spinat & \multicolumn{7}{|l|}{$<0,35$} \\
\hline Gluten & 75,93 & \multicolumn{5}{|l|}{5} & Kartoffel & \multicolumn{7}{|l|}{$<0,35$} \\
\hline \multicolumn{7}{|c|}{ Getreide glutenfrei } & Paprikaschote & \multicolumn{7}{|l|}{$<0,35$} \\
\hline Amaranth & $<0,35$ & & & & & & Tomate & 0,53 & 1 & & & & & \\
\hline Quinoa & 0,70 & \multicolumn{5}{|c|}{2} & Karotte & \multicolumn{7}{|l|}{$<0,35$} \\
\hline Hirse & 0,65 & \multicolumn{5}{|l|}{1} & Sellerie & \multicolumn{7}{|l|}{$<0,35$} \\
\hline Mais & $<0,35$ & & Avocado & \multicolumn{6}{|c|}{$0,5 7 \longdiv { 1 }$} & \\
\hline Buchweizen & $<0,35$ & & & & & & Gemüse-Pool & 0,60 & 1 & & & & & \\
\hline Reis & 1,51 & \multicolumn{5}{|c|}{2} & \multicolumn{8}{|l|}{ Hülsenfrüchte } \\
\hline Fleisch, Fisch, & & & & & & & Sojabohne & $<0,35$ & & & & & & \\
\hline Hühnerei & 24,51 & 4 & & & & & Grüne Bohne & 0,67 & 1 & & & & & \\
\hline Rind & $<0,35$ & & & & & & Erdnuss & 0,54 & 1 & & & & & \\
\hline Fische-Pool & $<0,35$ & & & & & & Hülsenfrüchte-Pool & $<0,35$ & & & & & & \\
\hline Fleisch-Pool & $<0,35$ & & & & & & Nüsse, Samen & & & & & & & \\
\hline Obst & & & & & & & Haselnuss & 18,80 & 4 & & & & & \\
\hline Kiwi & $<0,35$ & & & & & & Sonnenblumenkerne & $<0,35$ & & & & & & \\
\hline Weintraube & 0,44 & 1 & & & & & Sesam & $<0,35$ & & & & & & \\
\hline Banane & $<0,35$ & & & & & & Mandel & $>100,00$ & 6 & & & & & \\
\hline Apfel & 0,40 & 1 & & & & & Nüsse+Samen-Pool & 0,67 & 1 & & & & & \\
\hline
\end{tabular}

Abb. 4 Häufige Allergene.

4) Maßhalten mit säurehaltigen Nahrungsmitteln:

Nahrungssäuren, insbesondere Obstsäuren, führen zu einem Reizzustand der Darmschleimhaut. Nahrungssäuren lassen entzündliche Prozesse, Haut- und Schleimentzündungen sowie allergische Entzündungen, nicht zur Ruhe kommen. Die Säuren (Natrium- und Kaliumzitrate) verbinden sich mit Kalzium aus den Schleimhautzellen zu biologisch-inaktiven Salzen (Kalziumzitrat). Dadurch wird der Darmschleimhaut biologisch-aktives Kalzium entzogen. Es entsteht ein versteckter Kalziummangel, eine Verschiebung des Elektrolytgleichgewichts in den Membranen, es kommt zu Resoptionsstörungen und Entzündungen. Durch hohen Säuregehalt zeichnen sich aus: alles Obst, insbesondere Apfelsinen, Zitronen, Grapefruits, Beeren, alle Obstsäfte und Weine, dann Tomaten, Spinat, Salate, Gemüse und Gemüsesäfte. Es ist immer wieder eindrucksvoll, wie gerade das atopische Ekzem, Sinusitiden und Bronchitiden durch Obstkarenz zur Ruhe kommen.

\section{5) Smoothies sind Lebensnahrung und sollen „geges- sen" werden:}

Für eine ausreichende Vitamin- und Mineralienzufuhr empfehlen sich milde säurearme grüne Presssäfte aus einem Slowjuicer oder Entsafter: z. B. 100-150 ml Karottensaft, der ein besonders säurearmer, aber hochwertiger Vitamin- und Mineralstoffspender ist.
6) Eiweißarme Ernährung: So viel wie nötig, aber so wenig wie möglich:

Große Eiweißmengen unterhalten Entzündungsprozesse und doch muss das Angebot gerade an essenziellen Aminosäuren ausreichend sein. Daher lautet die Empfehlung, kleine Mengen unterschiedlicher Quellen über den Tag verteilt zu essen - das komplettiert das Angebot der essenziellen Aminosäuren und senkt den Gesamtbedarf. Insgesamt reichen $0,6 \mathrm{~g}$ Eiweißzufuhr pro kg Körpergewicht aus. Voraussetzung ist, dass die Mikroflora des Darms intakt ist und die Nahrungseiweiße nicht durch eine vermehrte proteolytische Fäulnisflora verloren gehen.

\section{7) Allergene und Immuntrigger meiden:}

Die häufigsten Allergene aus Nahrungsmitteln sind Eier, Nüsse, Mandeln, Kuhmilch, Gluten, Soja (>Abb. 4). Da es sich bei diesen Lebensmitteln um sog. Grundnahrungsmittel handelt und sie in vielen Nahrungsmitteln versteckt enthalten sind, empfiehlt sich eine möglichst genaue Testung folgender Immunreaktionen: IgE, IgG1-3 und IgG4, Antigliadine, evtl. Antitranglutaminase, um dann die individuellen Trigger gezielt zu eliminieren.

Reagieren Patienten auf sehr viele Nahrungseiweiße allergisch und müssen diese aus der täglichen Ernährung gestrichen werden, kann eine zeitweise Supplementierung mit hochwertigen reinen essenziellen Aminosäuren sinnvoll sein. 
\Tab. 1 Beispiel für einen Tagesspeiseplan. Als Speiseöle eignen sich Leinöl, Fischöl, Butter, Ghee.

\section{Frühstück}

Getreidebrei aus feinen Hirse-, Hafer-, Reisflocken mit Wasser gekocht

oder:

glutenfreies fein ausgemahlenes

helles Brot mit Butter/Ghee

dazu Öl oder Butter (ggf. Ghee) oder bei Kuhmilchverträglichkeit auch Sahne ggf. etwas süßen mit Zucker oder Dicksaft

oder:

ggf. mit Ei und Aufstrich, Schaf- oder

Ziegenkäse, Honig

\begin{tabular}{|l|l|l|l|}
\hline zwischendurch & Mittagessen & zwischendurch & Abendessen \\
\hline $\begin{array}{l}\text { Schaf- oder } \\
\text { Ziegenkäse }\end{array}$ & Fleisch/Fisch & $\begin{array}{l}\text { Kraftbrühe/ } \\
\text { Gemüsebrühe }\end{array}$ & $\begin{array}{l}\text { Fisch, Eier - auch kalt: } \\
\text { Räucherlachs, -Forelle... } \\
\text { oder: } \\
\text { Gemüsesuppe }\end{array}$ \\
\hline $\begin{array}{l}\text { Banane / ein } \\
\text { milder Apfel }\end{array}$ & $\begin{array}{l}\text { Kartoffeln oder } \\
\text { mildes Gemüse z. B. } \\
\text { Wurzelgemüse, weicher }\end{array}$ & $\begin{array}{l}\text { glutenfreier } \\
\text { Pudding }\end{array}$ & $\begin{array}{l}\text { Kartoffeln, Gemüse }(15 \mathrm{gH}) \text { oder: } \\
\text { glutenfreies helles Brot mit Butter/ } \\
\text { Ghee/Speiseölen }\end{array}$ \\
\hline
\end{tabular}
weißer Reis
IgG4 Nahrungsmittelscreen

\begin{tabular}{|c|c|c|c|c|c|c|c|}
\hline \multirow[t]{2}{*}{ Test } & \multirow{2}{*}{$\begin{array}{c}\text { Ergebnis } \\
\text { kU/I }\end{array}$} & \multicolumn{6}{|c|}{ Normbereich } \\
\hline & & 1 & 2 & 3 & 4 & 5 & 6 \\
\hline \multicolumn{8}{|c|}{ Getreide glutenhaltig } \\
\hline Dinkel & 80,68 & 5 & & & & & \\
\hline Hafer & 8,22 & 3 & & & & & \\
\hline Roggen & 26,81 & 4 & & & & & \\
\hline Weizen & 62,33 & 5 & & & & & \\
\hline Gluten & 40,74 & 4 & & & & & \\
\hline \multicolumn{8}{|c|}{ Getreide glutenfrei } \\
\hline Amaranth & 10,46 & 3 & & & & & \\
\hline Quinoa & 4,80 & 3 & & & & & \\
\hline Hirse & 15,10 & 3 & & & & & \\
\hline Mais & 19,47 & 4 & & & & & \\
\hline Buchweizen & 11,25 & 3 & & & & & \\
\hline Reis & 24,05 & 4 & & & & & \\
\hline
\end{tabular}

Abb. 5 Beispiel für einen Nahrungsmittelscreen.

Eine besondere Rolle bei allergisch-entzündlichen Erkrankungen nimmt die Kuhmilch ein. Die darin enthaltenen Wachstumsfaktoren und Hormone unterhalten entzündliche Vorgänge und sind deshalb grundsätzlich zu meiden.

Gluten und Gliadine, die Proteine aus Weizen und anverwandten Getreiden, sind ebenfalls zu meiden oder zumindest stark einzuschränken. Die entstehenden Eiweiß-Zucker-Verbindungen (Advanced Glycation Endproducts, AGEs) lassen Inflammationen und übermäßige Zytokinreaktionen nicht zur Ruhe kommen. Insbesondere die Vollkornprodukte enthalten darüber hinaus Amylase-TrypsinInhibitoren, die die Verdauung und Aufschlüsselung der Nahrung erschweren bis unmöglich machen. Das bedeutet, dass vermehrt hochmolekulare Proteine im distalen Dünndarm erscheinen und dort Immunreaktionen auslösen können.
Glutenhaltige Getreide dürfen aber nicht einfach durch glutenfreie Lebensmittel ersetzt werden! Hirse, Amaranth, Quinoa sind zwar glutenfrei, doch auch gegen diese können Immunreaktionen, besonders IgG-Unverträglichkeiten, auftreten, da sie nur sehr schwer zu verdauen sind ( $\triangleright$ Abb. 5). Bei Stuhlinspektionen sind die groben Körner immer wieder zu beobachten.

8) Kohlenhydratärmere Nahrungsauswahl - unterschiedliche Kohlenhydrate (Getreide, Kartoffeln und Gemüse) über den Tag verteilen - Eiweißträger nach individueller immunologischer Verträglichkeit

Ein Beispiel für einen Tagesspeiseplan ist in \ Tab. 1 aufgeführt.

\section{9) Mikrobiologische Therapie}

Eine individuell angepasste Mikrobiologische Therapie v. a. mit differenzierten Stämmen der Lactobazillen und Bifidobakterien unterstützt die Genesung. Autolysate aus Kolibakterien haben sich als hocheffiziente immunmodulatorische Therapie bewährt.

\section{Verlauf der Patientenfälle}

\section{Marie W. 10 Jahre}

Oberstes und erstes Ziel bei Marie war die Reduktion der Gärungsdyspepsie! Dazu musste Marie zunächst lernen, gut zu kauen, langsam und achtsam zu essen. Die Nahrung wurde weich gekocht: keine Rohkost, keine Körner oder ungekochte Getreideflocken. Weiterhin Kuhmilchund Eikarenz, aber auch glutenhaltige Lebensmittel musste Marie meiden. Zur Deckung des Eiweißbedarfs sollte sie zartes Fleisch und Fisch in den Alltag integrieren.

Schwierigkeit: Maries Mutter sah es ein, dass Fleisch und Fisch in den Alltag integriert werden musste, konnte es aber selbst in der Küche nicht zubereiten. Sie griff also vermehrt auf Fertigprodukte wie Würste, Salami oder Dosenfisch zurück, oder verwies auf die Schulküche zweimal die 


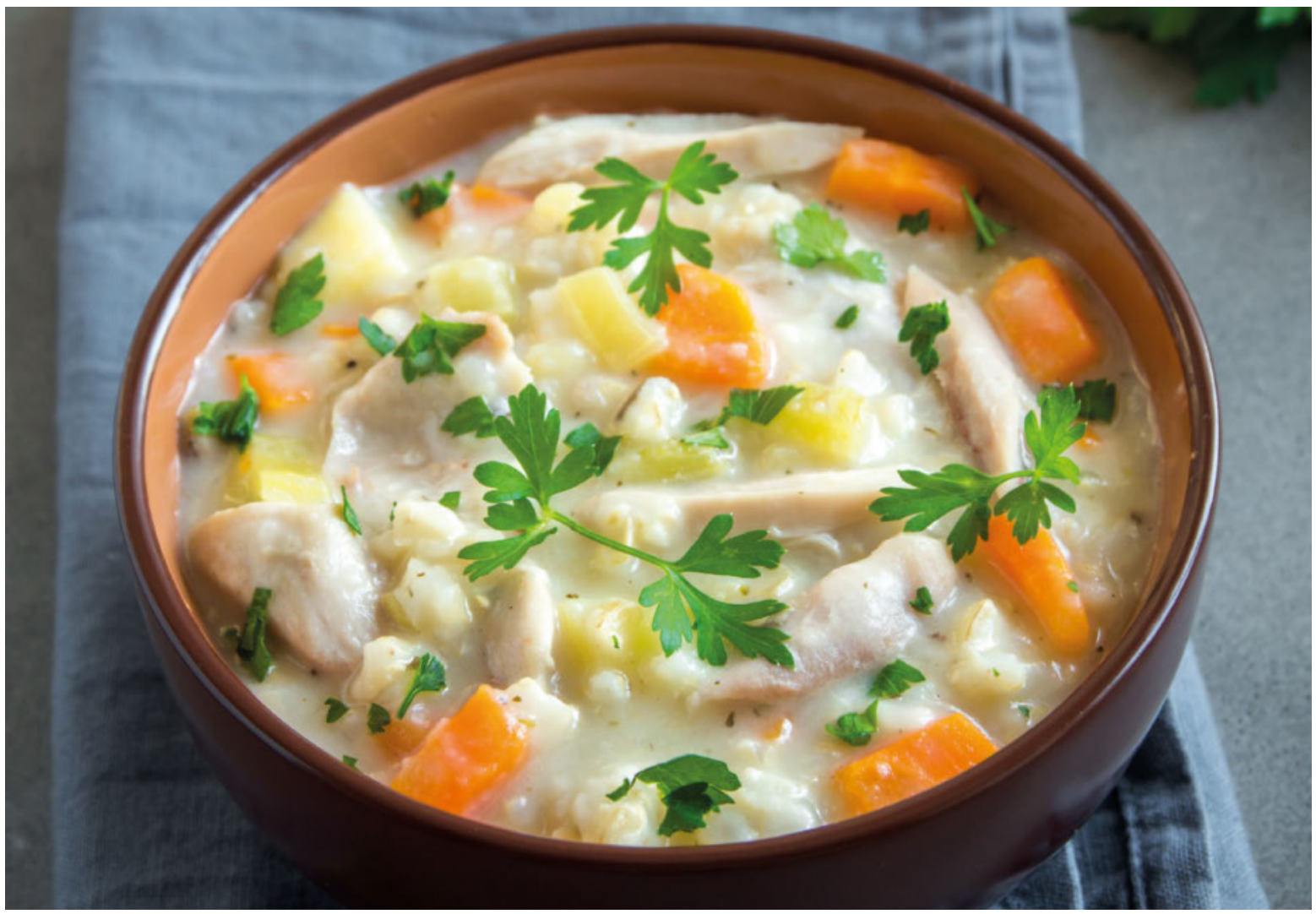

Abb. 6 Genauso wichtig wie die Auswahl der Nahrungsmittel ist die Art der Zubereitung: Gut durchgegarte Speisen erleichtern eine gute Verdauung. @ mizina/Adobe Stock

Woche. Nach intensiven Gesprächen gelang es dann, in der Familie den Weg zu finden: Maries Vater fand die Zeit und Freude daran, Fleisch und Fisch zuzubereiten.

Ernährung leicht verdaulich: morgens isst sie meistens einen feinen Reis- oder Hirsebrei mit Wasser gekocht und Butter - mit Zimt und wenig Zucker, am Vormittag etwas Schaf- oder Ziegenkäse mit einem kleinen Stück Gurke (in der Schule) mittags Fleisch oder Fisch ca. 50-80 g meistens mit Kartoffeln und Karotten- oder Zucchinigemüse, am Nachmittag dann einige glutenfreie feine Kekse, eine halbe Banane, abends isst sie wenig: eine Suppe, oder auch mal ein Brot mit Butter.

Mikrobiologische Therapie: Beginnend mit Prosymbioflor (Autolysat aus Enterokokken und E.coli) für 6 Wochen zur Immunregulation. Omnibiotic panda $1 \times$ tägl. (Lactobazillen, Bifidobakterien) für 6 Monate. Nach 2 Monaten Colibiogen für Kinder (zur weiterführenden Immunmodulation).

Verlauf: Nach einem Monat deutliche Entspannung im Darmbereich. Bauch weich und nur wenig druckdolent. Das Bindegewebe strafft sich, die Konzentrationsfähigkeit steigt. Die Haut ist frei und sie reagiert nicht mehr so stark auf Ei. Kleine Mengen (z. B. auf einem Geburtstagsfest) riefen keine Hautreaktionen mehr hervor.

\section{Lisa P. 52 Jahre}

Auch Frau P. lernte vor allem neu zu kauen. Die Nahrung haben wir auf 6 Mahlzeiten ausgewogen verteilt: Kein Vollkorn! Zu Beginn kein Salat, nach 4 Wochen wenige zarte Blätter mittags. Jeweils ein Eiweißträger und eine kleine Beilage. Keine histaminhaltigen Lebensmittel.

Verlauf: Nach wenigen Tagen verschwanden die Blähungen, der Stuhl normalisierte sich zusehends. Darüber hinaus bekam die Patientin vier Colon-Hydro-Therapien zur Ausleitung und Immunregulation und regelmäßig F.X. Mayr-Darmmassagen, die sie auch selbstständig zuhause durchzuführen lernte. Zur Unterstützung erhielt sie eine Mikrobiologische Therapie mit Lactobazillen und Bifidobakterien (Omnibiotic stress repair) und Injektionen von Colibiogen zur Immunmodulation. Die Urtikaria blasste innerhalb von 14 Tagen vollständig ab, inzwischen kann sie wieder kleine Mengen an Alkohol und altem Käse ohne Reaktionen vertragen.

\section{Silke F. 43 Jahre}

Therapeutisches Vorgehen entsprechend den allgemeinen Richtlinien: Das Vordringlichste war das Neu-Erlernen des langsamen Essens. Die Patientin nahm an einer KauSchulung mit anderen Patienten teil und lernte so eine neue Ess- und Schlucktechnik. Die Mahlzeiten wurden kleiner, der Bauch flacher. 
Ernährung: Kuhmilcheiweißkarenz und Reduktion von Obstsäuren auf ein mildes Obst pro Tag. Glutenfreie Getreide für 4 Monate (mit Ausnahme an Familienfesten max. einmal pro Woche).

Mikrobiologische Therapie: 4 Wochen Autolysat von Enterokokken und E. coli-Bakterien, 6 Monate: Lebendkeime Lactobazillen, Bacteroides und Bifidobakterien, anschließend Autolysat aus E.coli-Bakterien für 4 Monate zur Immunstabilisierung.

Verlauf: Nach 4 Wochen deutliche Reduktion und Abheilung des rissigen Handekzems. Nach 4 Monaten vollständige Abheilung der Handflächen - keine anderen Ekzemherde mehr vorhanden.

Das Ganze fiel der Patientin nicht schwer, denn sie hatte zudem auch einen Zugewinn an Kraft und Vitalität.

\section{Konsequenzen für die ärztliche Praxis}

Kaum ein anderes therapeutisches Verfahren greift so grundlegend und tief in den Genesungsprozess ein wie die Sanierung des Verdauungssystems. Sie ist Dreh- und Angelpunkt jeder naturheilkundlichen Therapie. Wenn die intestinale Intoxikation und bakterielle Dyspepsie ausheilt, kommen das Immunsystem und alle entzündlichen Reaktionen zur Ruhe.

Begleitend dazu können Spülungen des Verdauungsorgans (Bittersalze, hohe Einläufe oder eine Colon-Hydro-Therapie) und eine forcierte Ausleitung über die Atemluft (Bewegung) oder die Niere (Trinken, Nieren- und Blasentee) die Haut entlasten.

Darüber hinaus kann und muss das ganze Spektrum der naturheilkundlichen Verfahren genutzt werden: Abgelagerte Stoffwechselschlacken müssen behutsam mobilisiert und über Haut und Nieren ausgeschieden werden. Gerade bei Allergien haben sich körperwarme Langzeitbäder, Sauna und maßvoller Sport bewährt. Wöchentliche Überwärmungsbäder greifen tiefgreifend in die Regulation des Immunsystems ein. Bei regelmäßiger Anwendung kommen überschießende allergische Reaktionen nachhaltig zur Ruhe.

\section{Interessenkonflikt}

Die Autorin gibt an, dass kein Interessenkonflikt besteht.
Autorin

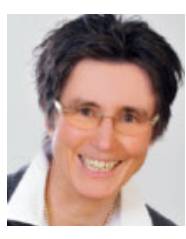

Dr. med. Monika Pirlet-Gottwald

Monika Pirlet-Gottwald ist in eigener Praxis niedergelassen. Behandlungsschwerpunkte: Naturheilkunde, physikalische Therapie, Ernährungsmedizin. Vizepräsidentin des Zentralverbands für Naturheilverfahren und Regulationsmedizin (ZAEN) e. V.

\section{Korrespondenzadresse}

\author{
Dr. med. Monika Pirlet-Gottwald \\ Ärztin - Naturheilkunde \\ Ernährungsmedizinerin \\ Waisenhausstr. 52a \\ 80637 München \\ E-Mail: pirlet-gottwald@t-online.de
}

\section{Literatur}

[1] Gonder U, Wort N. Mehr Fett! - Warum wir mehr Fett brauchen, um gesund und schlank zu sein. Lünen: Systemed; 2010

[2] Hauss W. Unspezifische Mesenchymreaktion und die primärchronischen Mesenchymkrankheiten. Dt. Ärztebl. 1992; 89 (10): A792-800

[3] Pirlet K. Intestinale Autointoxikation und Intestinales Immunsystem. In: Zilch M], Uecker D, Hrsg. Immunologie. Neckarsulm: Jungjohann; 1990: 71-80

[4] Pirlet-Gottwald M. Patientenanleitung zur Ernährungsweise bei Verdauungs- und Stoffwechselstörungen. zaenmagazin 2013; 3: 22-29

[5] Pirlet-Gottwald M. Haut und Schleimhaut: Ernährung als Therapie bei Hauterkrankungen. EHK 2016; 65: 278-283

[6] Rauch E. Lehrbuch der Diagnostik nach F.X. Mayr. Heidelberg: Haug; 1994

[7] Schmidt R. Schnitzer S. Allergie und Mikrobiota. Stuttgart: Haug; 2017

[8] Schnitzer S. Bestandteile glutenhaltiger Getreidesorten und ihr Einfluss auf Stoffwechsel und Immunsystem, Grenzfläche und Milieu. OM \& Ernährung 2016; 157: 30-36

[9] Schütz B, Muss C. Nahrungsmittelunverträglichkeiten. Komplementäre und integrative Medizin 2007; 12: 30-37

[10] Wendt L. Die Eiweißspeichererkrankungen. Heidelberg: Haug; 1984

\section{Bibliografie}

DOI https://doi.org/10.1055/s-0044-100752 EHK 2018; 67: 22-30

(c) MVS Medizinverlage Stuttgart GmbH \& Co. KG ISSN 0014-0082 